\title{
Elliptic Problems with Nonhomogeneous Differential Operators and Multiple Solutions
}

\author{
Dumitru Motreanu and Patrick Winkert
}

\begin{abstract}
The present survey aims to report on recent advances in the study of nonlinear elliptic problems whose differential part is expressed by a general operator in divergence form. The pattern of such differential operator is the $p$-Laplacian $\Delta_{p}$ with $1<p<+\infty$. More general operators can be considered, possibly having completely different properties, for instance not satisfying any homogeneity requirement. A major objective of our work is to provide existence theorems of multiple solutions for boundary value problems governed by such general operators. In this direction, a three nontrivial solutions theorem is presented. In the case of problems determined by the $p$-Laplacian, we give a theorem ensuring the existence of at least four nontrivial solutions. Moreover, a complete sign information is available: two positive solutions, a negative solution and a nodal (sign-changing) solution. Finally, we provide a theorem guaranteeing the existence of a positive solution for a problem involving the $(p, q)$-Laplacian operator $\Delta_{p}+\Delta_{q}$, with $1<q<p$, and a nonlinearity depending on the solution and its gradient.
\end{abstract}

Keywords Nonlinear elliptic boundary value problem · Nonhomogeneous differential operator $\cdot p$-Laplacian · Eigenvalue problem - Multiple solutions · Critical point · Minimizer $\cdot$ Regularity $\cdot$ Maximum principle

\section{Introduction}

In this survey we focus on nonlinear elliptic boundary value problems with Dirichlet and Neumann boundary conditions presenting recent existence results of multiple solutions with precise sign information such as positive, negative and nodal (i.e., signchanging) solutions. Specifically, we address some advances related to the nonlinear

\footnotetext{
D. Motreanu $(\bowtie)$

Département de Mathématiques, Université de Perpignan, Avenue Paul Alduy 52,

66860 Perpignan, France

e-mail: motreanu@univ-perp.fr

P. Winkert

Technische Universität Berlin, Institut für Mathematik, Straße des 17. Juni 136,

10623 Berlin, Germany

e-mail: winkert@math.tu-berlin.de
} 
Dirichlet boundary value problem

$$
\begin{cases}-\operatorname{div} A(x, \nabla u)=f(x, u) & \text { in } \Omega \\ u=0 & \text { on } \partial \Omega\end{cases}
$$

and the nonlinear Neumann boundary value problem

$$
\begin{cases}-\operatorname{div} A(x, \nabla u)=f(x, u) & \text { in } \Omega, \\ \frac{\partial u}{\partial n}=0 & \text { on } \partial \Omega,\end{cases}
$$

where $n$ denotes the outward unit normal vector on $\partial \Omega, 1<p<+\infty$ is a given number, and $\Omega \subset \mathbb{R}^{N}$ is a bounded domain with $C^{2}$ boundary $\partial \Omega$. For a later use we denote by $\bar{\Omega}$ the closure of $\Omega$ in $\mathbb{R}^{N}$.

The nonlinearity in the right-hand side of the elliptic equation in (1) and (2) is required to satisfy the following assumption:

(F) $f: \Omega \times \mathbb{R} \rightarrow \mathbb{R}$ is a Carathéodory function, which means that $f(x, t)$ is measurable in $x$ and continuous in $t$, with $f(x, 0)=0$ for a.e. $x \in \Omega$, such that the subcritical growth condition for $f(x, \cdot)$ holds

$$
|f(x, t)| \leq C\left(1+|t|^{r-1}\right) \quad \text { for every } t \in \mathbb{R} \text {, a.e. } x \in \Omega,
$$

where $C>0$ and $1 \leq r<p^{*}$ are constants. Here $p^{*}$ denotes the critical Sobolev exponent, that is

$$
p^{*}= \begin{cases}\frac{N p}{N-p} & \text { if } p<N \\ +\infty & \text { if } p \geq N\end{cases}
$$

The left-hand side of (1) and (2) is expressed in the form of divergence $\operatorname{div} A(x, \nabla$ $(\cdot))$ and is also nonlinear. Therein we have a continuous map $A: \bar{\Omega} \times \mathbb{R}^{N} \rightarrow \mathbb{R}^{N}$ satisfying the growth condition

$$
|A(x, y)| \leq C\left(1+|y|^{p-1}\right) \quad \text { for all }(x, y) \in \bar{\Omega} \times \mathbb{R}^{N},
$$

with a constant $C>0$. In the above statement the number $1<p<+\infty$ is the same as the one in assumption $(F)$.

We say that $u \in W_{0}^{1, p}(\Omega)$ is a (weak) solution of problem (1) if

$$
\int_{\Omega} A(x, \nabla u) \cdot \nabla v d x=\int_{\Omega} f(x, u) v d x \text { for all } v \in W_{0}^{1, p}(\Omega) .
$$

Similarly, we say that $u \in W^{1, p}(\Omega)$ is a (weak) solution of problem (2) if

$$
\int_{\Omega} A(x, \nabla u) \cdot \nabla v d x=\int_{\Omega} f(x, u) v d x \text { for all } v \in W^{1, p}(\Omega) .
$$

We note that owing to the above growth conditions, the integrals involved in (3) and (4) exist. 
In the following we describe the topics studied in this chapter. Section 2 discusses the hypotheses on the differential operator in (1) and (2) and important properties of it. We stress that we are dealing with a general notion, for instance no homogeneity assumptions are supposed. We point out that under suitable assumptions, the problems (1) and (2) have a variational structure. Looking for the critical points of the Euler functionals associated to problems (1) and (2), which coincide with the weak solutions of these problems, the obvious candidates are the local minimizers. In this respect, Sect. 3 sets forth the celebrated relation of $C^{1}$-minimizers versus $W^{1, p}$-minimizers for problem (2) in the case of our general differential operator determined by the possibly nonhomogeneous map $A(x, y)$.

As noted above, a major difficulty in handling problems (1) and (2) is the lack of homogeneity for operator $A(x, y)$. This is seen for example in the study of the spectrum of the corresponding differential operator $-\operatorname{div} A(x, \nabla u)$ driving the principal part in the problems (1) and (2). In Sect. 4, in order to overcome the lack of homogeneity, asymptotic $(p-1)$-homogeneity at zero and infinity conditions for the nonhomogeneous operator $A(x, \cdot)$ are introduced, under which a basic result on the spectrum of the operator $-\operatorname{div} A(x, \nabla u)$ is stated. Next, in Sect. 5 it is presented a multiplicity theorem ensuring the existence of at least three nontrivial solutions for problem (2). Two of the solutions are of opposite constant signs and are obtained by minimization, whereas the existence of the third nontrivial solution is deduced through the application of the mountain pass theorem. The proof strongly relies on the relation involving $C^{1}$-minimizers versus $W^{1, p}$-minimizers.

More insight on multiple solutions with complete sign information for problem (1) is obtained in the case where $\operatorname{div} A(x, \nabla u)$ is the $p$-Laplacian operator. Here a striking particularity of the studied problem is that the right-hand side nonlinearity contains a $(p-1)$-sublinear term (usually, called "concave term") $\beta(x)|u(x)|^{q-2} u(x)$ with $\beta \in L^{\infty}(\Omega) \backslash\{0\}, \beta \geq 0$, and $1<q<p$. In Sect. 6 we state a result guaranteeing the existence of at least two positive solutions provided $\|\beta\|_{\infty}$ is sufficiently small. Section 7 is devoted to the existence of opposite constant sign extremal solutions, which means the smallest positive solution and the biggest negative solution. This is done by applying the method of sub-supersolutions. Section 8 contains our main result on multiple solutions with precise sign information, which ensures the existence of four nontrivial solutions: two positive, one negative and one nodal (sign changing). The proof is based on the existence of extremal solutions of opposite constant sign exploiting the observation that every nontrivial solution situated between opposite constant sign extremal solutions is necessarily nodal. This technique originates in [4] (see also [5]).

Finally, a new problem is formulated in Sect. 9 which is conducted by the $(p, q)$ Laplacian operator $\Delta_{p}+\Delta_{q}$, with $1<q<p$, and whose right-hand side depends on the solution and its gradient. The dependence of the nonlinearity of the gradient of the solution prevents the problem from having a variational structure. Our result ensures the existence of a positive solution. The approach combines approximation, fixed point argument and an adequate comparison principle. 


\section{Properties of the Differential Operator in (1) and (2)}

The map $A$ entering the left-hand side of problems (1) and (2) is assumed to satisfy the following conditions:

(H) $A(x, y)=a(x,|y|) y$, where $a(x, t)>0$ for all $(x, t) \in \bar{\Omega} \times(0,+\infty)$, and

(i) $A \in C^{0}\left(\bar{\Omega} \times \mathbb{R}^{N}, \mathbb{R}^{N}\right) \cap C^{1}\left(\bar{\Omega} \times\left(\mathbb{R}^{N} \backslash\{0\}\right), \mathbb{R}^{N}\right)$;

(ii) There exists a constant $C_{1}>0$, such that

$$
\left|D_{y} A(x, y)\right| \leq C_{1}|y|^{p-2} \quad \text { for every } x \in \bar{\Omega} \text {, and } y \in \mathbb{R}^{N} \backslash\{0\} ;
$$

(iii) There exists a constant $C_{0}>0$ such that $D_{y} A(x, y) \xi \cdot \xi \geq C_{0}|y|^{p-2}|\xi|^{2} \quad$ for every $x \in \bar{\Omega}, y \in \mathbb{R}^{N} \backslash\{0\}$, and $\xi \in \mathbb{R}^{N}$

(iv) There exists a constant $C_{2}>0$ such that

$$
\left|D_{x} A(x, y)\right| \leq C_{2}\left(1+|y|^{p-1}\right) \quad \text { for every } x \in \bar{\Omega} \text {, and } y \in \mathbb{R}^{N} \backslash\{0\}
$$

(v) There exist constants $C_{3}>0$ and $1 \geq t_{0}>0$ such that

$$
\begin{array}{r}
\left|D_{x} y A(x, y)\right| \leq C_{3}|y|^{p-1}(-\log |y|) \quad \text { for every } x \in \bar{\Omega}, \\
y \in \mathbb{R}^{N} \backslash\{0\} \text { with } 0<|y|<t_{0} .
\end{array}
$$

The notation $D_{y} A$ means the differential of the mapping $A(x, y)$ with respect to the variable $y \in \mathbb{R}^{N}$, and here $1<p<+\infty$ is given in assumption $(F)$. Notice that if $A$ does not depend on $x \in \Omega$, then conditions (H) (iv), (v) are automatically satisfied.

We provide some examples of maps $A$ complying with hypothesis $(H)$.

Example 2.1 Let $\theta \in C^{1}(\bar{\Omega})$ with $\theta>0$. Then the following mappings fulfil the conditions stated in $(H)$.

(i) For $1<p<+\infty$ : $A(x, y)=\theta(x)|y|^{p-2} y$;

(ii) For $p \geq 2$ : $A(x, y)=\theta(x)\left(|y|^{p-2} y+\ln \left(1+|y|^{p-2}\right) y\right)$;

(iii) For $1<\tau \leq p \leq q$ and $\tau \neq 2$ :

$$
A(x, y)= \begin{cases}\theta(x)\left(|y|^{p-2} y+|y|^{q-2} y\right) & \text { if }|y| \leq 1 \\ \theta(x)\left(|y|^{p-2} y+\frac{q-2}{\tau-2}|y|^{\tau-2} y-\frac{q-\tau}{\tau-2} y\right) & \text { if }|y|>1\end{cases}
$$

(iv) The weighted sum of $p$-Laplacian and a generalized mean curvature operator:

$$
A(x, y)=\theta(x)\left(|y|^{p-2} y+c \frac{|y|^{p-2} y}{1+|y|^{p}}\right)
$$

with $0<c<4 p(p-1)$ if $1<p<2$ and $0<c<\frac{4 p}{(p-1)^{2}}$ if $p \geq 2$. 
The form of the operator $A$ allows us to build a variational setting for problems (1) and (2). To this end, we note that the continuity of $A$ guaranteed by hypothesis $(H)$ (i) implies that the function $(x, t) \mapsto a(x, t) t$ is continuous for all $x \in \bar{\Omega}$ and $t \geq 0$. This can be seen from the equality

$$
A(x,(t, 0, \ldots, 0))=(a(x, t) t, 0, \ldots, 0) \text { for all } x \in \bar{\Omega}, t>0 .
$$

Therefore for every $x \in \bar{\Omega}$ and $y \in \mathbb{R}^{N}$ it is well defined:

$$
G(x, y):=\int_{0}^{|y|} a(x, t) t d t
$$

A straightforward computation shows that the gradient $\nabla_{y} G(x, y)$ with respect to the variable $y \in \mathbb{R}^{N}$ is given by

$$
\nabla_{y} G(x, y)=A(x, y) \text { for all } x \in \bar{\Omega} \text { and } y \in \mathbb{R}^{N} .
$$

Moreover, due to hypothesis $(H)$ (iii), $G(x, y)$ is convex in $y$ for all $x$ and satisfies:

$$
A(x, y) \cdot y \geq G(x, y) \text { for all }(x, y) \in \bar{\Omega} \times \mathbb{R}^{N} .
$$

As a consequence of $(H)$ and by means of (5) we have the following proposition that summarizes some significant facts regarding the operator $A$ and the corresponding potential $G$.

Proposition 2.1 Let hypothesis $(H)$ be satisfied. Then there hold:

(i) The map $y \mapsto A(x, y)$ is maximal monotone and strictly monotone for all $x \in \bar{\Omega}$.

(ii)

$$
|A(x, y)| \leq \frac{C_{1}}{p-1}|y|^{p-1} \quad \text { for all }(x, y) \in \bar{\Omega} \times \mathbb{R}^{N} .
$$

(iii)

$$
A(x, y) \cdot y \geq \frac{C_{0}}{p-1}|y|^{p} \quad \text { for all }(x, y) \in \bar{\Omega} \times \mathbb{R}^{N} .
$$

$$
\frac{C_{0}}{p(p-1)}|y|^{p} \leq G(x, y) \leq \frac{C_{1}}{p(p-1)}|y|^{p} \quad \text { for all }(x, y) \in \bar{\Omega} \times \mathbb{R}^{N} .
$$

The following result mentioning an essential property of the differential operator $\operatorname{div} A(x, \nabla(\cdot))$ corresponding to the map $A$ was proven in [20, Proposition 10]. This result is a key tool in checking the Palais-Smale condition for the Euler functional associated to problems (1) and (2).

Proposition 2.2 Assume hypothesis $(H)$. Then the map $V: W^{1, p}(\Omega) \rightarrow$ $\left(W^{1, p}(\Omega)\right)^{*}$ defined by

$$
\langle V(u), v\rangle=-\langle\operatorname{div} A(x, \nabla u), v\rangle=\int_{\Omega} A(x, \nabla u) \cdot \nabla v d x
$$


for all $u, v \in W^{1, p}(\Omega)$, is maximal monotone and has the $(S)_{+}$property, that is, any sequence $\left(u_{n}\right) \subset W^{1, p}(\Omega)$ such that $u_{n} \rightarrow u$ in $W^{1, p}(\Omega)$ and

$$
\limsup _{n \rightarrow \infty}\left\langle V\left(u_{n}\right), u_{n}-u\right\rangle \leq 0,
$$

for some $u \in W^{1, p}(\Omega)$, fulfills $u_{n} \rightarrow u$ in $W^{1, p}(\Omega)$.

Another useful related result is the following one whose proof is given in [23].

Proposition 2.3 Assume hypothesis $(H)$. For any number $\lambda>0$, let the mapping $T_{\lambda}: W^{1, p}(\Omega) \rightarrow\left(W^{1, p}(\Omega)\right)^{*}$ be defined by

$$
\left\langle T_{\lambda}(u), v\right\rangle=\int_{\Omega} A(x, \nabla u) \cdot \nabla v d x+\lambda \int_{\Omega}|u|^{p-2} u v d x .
$$

Then the inverse $T_{\lambda}^{-1}:\left(W^{1, p}(\Omega)\right)^{*} \rightarrow W^{1, p}(\Omega)$ of $T_{\lambda}$ exists and is continuous.

\section{$3 \quad C^{1}$-Minimizers Versus $W^{1, p}$-Minimizers for Problem (2)}

This section is devoted to the famous result dealing with the comparison of local $C^{1}$-minimizers and local $W^{1, p}$-minimizers for Euler functionals associated to some elliptic boundary value problems. Precisely, we set forth such a result in the case of our problem (2). A similar result holds for the Dirichlet problem (1) with $W_{0}^{1, p}(\Omega)$ in place of $W^{1, p}(\Omega)$.

Recall that the function $f: \Omega \times \mathbb{R} \rightarrow \mathbb{R}$ entering problem (2) is subject to hypothesis $(F)$ asserting the subcritical growth condition for $f$. Setting $F(x, t)=$ $\int_{0}^{t} f(x, s) d s$, we define the functional $\varphi: W^{1, p}(\Omega) \rightarrow \mathbb{R}$ by

$$
\varphi(u)=\int_{\Omega} G(x, \nabla u) d x-\int_{\Omega} F(x, u) d x,
$$

with the function $G$ introduced in Sect. 2. Under assumptions $(H)$ and $(F)$, the functional $\varphi$ is well defined on $W^{1, p}(\Omega)$ and is of class $C^{1}$. Moreover, its critical points coincide with the (weak) solutions of problem (2), that is, we have $\varphi^{\prime}(u)=0$ if and only if (4) holds true.

The main result of this section is the following.

Theorem 3.1 Assume that the conditions $(H)$ and $(F)$ are fulfilled. If $u_{0} \in W^{1, p}(\Omega)$ is a local $C^{1}(\bar{\Omega})$-minimizer of $\varphi$ in (6), that is, there exists $r_{0}>0$ such that

$$
\varphi\left(u_{0}\right) \leq \varphi\left(u_{0}+h\right) \quad \text { for all } h \in C^{1}(\bar{\Omega}) \text { with }\|h\|_{C^{1}(\bar{\Omega})} \leq r_{0},
$$

then $u_{0} \in C^{1}(\bar{\Omega})$ with $\frac{\partial u_{0}}{\partial n}=0$ on $\partial \Omega$, and it is a local $W^{1, p}(\Omega)$-minimizer of $\varphi$, that is, there exists $r_{1}>0$ such that

$$
\varphi\left(u_{0}\right) \leq \varphi\left(u_{0}+h\right) \quad \text { for all } h \in W^{1, p}(\Omega) \text { with }\|h\|_{W^{1, p}(\Omega)} \leq r_{1} .
$$




\section{Proof (sketch)}

Since $C^{1}(\bar{\Omega})$ is dense in $W^{1, p}(\Omega)$, it follows that $\varphi^{\prime}\left(u_{0}\right)=0$, or equivalently

$$
-\operatorname{div} A\left(x, \nabla u_{0}(x)\right)=f\left(x, u_{0}(x)\right) \text { in } \Omega, \frac{\partial u_{0}}{\partial n}=0 \text { on } \partial \Omega .
$$

Suppose that $u_{0}$ is not a local $W^{1, p}(\Omega)$-minimizer of $\varphi$. Because the functional $\varphi$ is sequentially weakly lower semicontinuous, for $\varepsilon>0$ small we can find $h_{\varepsilon} \in$ $\bar{B}_{\varepsilon}^{r}:=\left\{u \in W^{1, p}(\Omega):\|u\|_{L^{r}(\Omega)} \leq \varepsilon\right\}$ such that

$$
\varphi\left(u_{0}+h_{\varepsilon}\right)=\inf \left\{\varphi\left(u_{0}+u\right): u \in \bar{B}_{\varepsilon}^{r}\right\}<\varphi_{0}\left(u_{0}\right) .
$$

The Lagrange multiplier rule yields $\lambda_{\varepsilon} \leq 0$ with

$$
\varphi^{\prime}\left(u_{0}+h_{\varepsilon}\right)=\lambda_{\varepsilon}\left|h_{\varepsilon}\right|^{r-2} h_{\varepsilon}
$$

which results in

$$
-\operatorname{div} A\left(x, \nabla\left(u_{0}+h_{\varepsilon}\right)(x)\right)=f\left(x,\left(u_{0}+h_{\varepsilon}\right)(x)\right)+\lambda_{\varepsilon}\left|h_{\varepsilon}(x)\right|^{r-2} h_{\varepsilon}(x) \quad \text { in } \Omega .
$$

Setting $\tilde{A}(x, y):=A\left(x, \nabla u_{0}(x)+y\right)-A\left(x, \nabla u_{0}(x)\right)$, it is seen that $\tilde{A}$ satisfies

$$
-\operatorname{div} \tilde{A}\left(x, \nabla h_{\varepsilon}\right)=f\left(x, u_{0}+h_{\varepsilon}\right)-f\left(x, u_{0}\right)+\lambda_{\varepsilon}\left|h_{\varepsilon}\right|^{r-2} h_{\varepsilon} \quad \text { in } \Omega .
$$

Now we can show that there exists a constant $d_{0}>0$ such that $\left|\lambda_{\varepsilon}\right|\left|h_{\varepsilon}(x)\right|^{r-1} \leq d_{0}$ for all $x \in \Omega$ and $\varepsilon>0$ small.

At this point, in view of Lieberman [17, Theorem 2] there exist $\theta \in(0,1)$ and $M>0$ such that

$$
h_{\varepsilon} \in C^{1, \theta}(\bar{\Omega}) \text { and }\left\|h_{\varepsilon}\right\|_{C^{1, \theta}(\bar{\Omega})} \leq M \text { for all } \varepsilon>0 \text { small. }
$$

Exploiting that $C^{1, \theta}(\bar{\Omega})$ is embedded compactly in $C^{1}(\bar{\Omega})$, we infer that $u_{0}+h_{\varepsilon} \rightarrow$ $u_{0}$ in $C^{1}(\bar{\Omega})$ as $\varepsilon \rightarrow 0$. Consequently, assertion (7) yields $\varphi\left(u_{0}\right) \leq \varphi_{0}\left(u_{0}+h_{\varepsilon}\right)$ for $\varepsilon$ sufficiently small, which contradicts the choice of $h_{\varepsilon}$. This completes the proof.

Theorem 3.1 was proven in [20, Proposition 24] (see also [22, Theorem 3.1]). We emphasize that if $C_{0}=C_{1}=p-1$ in assumption $(H)$, the operator $V=$ $\operatorname{div} A(x, \nabla(\cdot)): W^{1, p}(\Omega) \rightarrow\left(W^{1, p}(\Omega)\right)^{*}$, with $A$ in hypothesis $(H)$, becomes the $p$ Laplacian operator on $W^{1, p}(\Omega)$. For $p$-Laplacian operator and Neumann problems, the result can be found in [26]. The counterpart result for Dirichlet problems in the case $p=2$ is due to Brezis and Nirenberg [3], which was extended to $1<p<+\infty$ by García Azorero, Peral Alonso, and Manfredi [10] (see also [14] for $p \geq 2$ ). For different extensions and related results we refer to $[2,8,12,15,16,21,31,32]$. Theorem 3.1 is very useful in the study of problem (2), especially for obtaining the existence of multiple solutions through variational methods, for example by using the mountain pass theorem, combined with the method of sub-supersolutions. 


\section{Asymptotically Homogeneous Case}

The eigenvalue problem with Dirichlet boundary condition for the nonlinear operator $\operatorname{div} A(x, \nabla(\cdot))$ means the existence of $\lambda \in \mathbb{R}$ for which

$$
\begin{cases}-\operatorname{div} A(x, \nabla u)=\lambda|u|^{p-2} u & \text { in } \Omega, \\ u=0 & \text { on } \partial \Omega,\end{cases}
$$

has a nontrivial solution. The eigenvalue problem $(E V ; \lambda)$ is hard because the operator $A(x, \cdot)$, so $\operatorname{div} A(x, \nabla(\cdot))$, is not homogeneous. Even in the case of the $p$-Laplacian $\Delta_{p}$, which is $(p-1)$-homogeneous, the spectrum is not completely known (it is known what is called the beginning of the spectrum of $-\Delta_{p}$ as can be seen in [7], see also [25, 24]).

In order to overcome the lack of homogeneity in $(E V ; \lambda)$ it was introduced in [23] an asymptotic $(p-1)$-homogeneity at zero condition for the nonhomogeneous operator $A(x, \cdot)$ :

(AH0) There exist a positive function $a_{0} \in C^{1}(\bar{\Omega}, \mathbb{R})$ and a continuous function $\tilde{a}_{0}(x, t)$ on $\bar{\Omega} \times[0,+\infty)$ such that

$$
\begin{aligned}
& A(x, y)=a_{0}(x)|y|^{p-2} y+\tilde{a}_{0}(x,|y|) y \quad \text { for all } x \in \Omega, y \in \mathbb{R}^{N}, \\
& \lim _{t \rightarrow 0^{+}} \frac{\tilde{a}_{0}(x, t)}{t^{p-2}}=0 \quad \text { uniformly in } x \in \bar{\Omega} .
\end{aligned}
$$

Under assumption $(A H 0)$, with the weight function $a_{0}$ we define

$$
\lambda_{1}\left(a_{0}\right):=\inf \left\{\int_{\Omega} a_{0}(x)|\nabla u|^{p} d x ;\|u\|_{p}=1\right\} .
$$

Also in order to overcome the lack of homogeneity in $(E V ; \lambda)$ it was introduced in [23] an asymptotic $(p-1)$-homogeneity at infinity condition for the nonhomogeneous operator $A(x, \cdot)$ :

$(A H)$ There exist a positive function $a_{\infty} \in C^{1}(\bar{\Omega}, \mathbb{R})$ and a continuous function $\tilde{a}(x, t)$ on $\bar{\Omega} \times \mathbb{R}$ such that

$$
\begin{aligned}
& A(x, y)=a_{\infty}(x)|y|^{p-2} y+\tilde{a}(x,|y|) y \quad \text { for all } x \in \Omega, y \in \mathbb{R}^{N}, \\
& \lim _{t \rightarrow+\infty} \frac{\tilde{a}(x, t)}{t^{p-2}}=0 \quad \text { uniformly in } x \in \bar{\Omega} .
\end{aligned}
$$

Under assumption $(A H)$, with the weight function $a_{\infty}$, we define

$$
\lambda_{1}\left(a_{\infty}\right):=\inf \left\{\int_{\Omega} a_{\infty}(x)|\nabla u|^{p} d x ;\|u\|_{p}=1\right\} .
$$

We cite from [23] the following result on the spectrum of the nonlinear operator $\operatorname{div} A(x, \nabla(\cdot))$. 
Theorem 4.1 Assume $(H),(A H 0),(A H)$ and that $\lambda_{1}\left(a_{0}\right) \neq \lambda_{1}\left(a_{\infty}\right)$. Then, for every $\lambda$ between $\lambda_{1}\left(a_{0}\right)$ and $\lambda_{1}\left(a_{\infty}\right)$, problem $(E V ; \lambda)$ has a nontrivial positive solution, therefore $\lambda$ is an eigenvalue of the operator $-\operatorname{div} A(x, \nabla(\cdot))$.

Theorem 4.1 is important because it shows that generally the spectrum of the operator $-\operatorname{div}(A(x, \nabla u))$ on $W_{0}^{1, p}(\Omega)$ is not discreet. However, Theorem 4.1 cannot be applied to the negative $p$-Laplacian $-\Delta_{p}$ on $W_{0}^{1, p}(\Omega)$ because in this case $\lambda_{1}\left(a_{0}\right)=\lambda_{1}\left(a_{\infty}\right)$.

\section{A Three Solutions Theorem}

In this section we present an existence and multiplicity result for the Neumann problem (2), on a bounded domain $\Omega \subset \mathbb{R}^{N}$ with a $C^{2}$-boundary $\partial \Omega$, involving the nonhomogeneous operator $A$ introduced in Sect. 2 . We suppose that the nonlinearity $f(x, u)$ in the right-hand side of the equation in (2) satisfies the following conditions:

$\left(H_{f}\right)_{1} f: \Omega \times \mathbb{R} \rightarrow \mathbb{R}$ is a Carathéodory function such that $f(x, 0)=0$ a.e. in $\Omega$ and

(i) $|f(x, s)| \leq a(x)+C|s|^{r-1}$ for a.a. $x \in \Omega$, all $s \in \mathbb{R}$, with $a \in L^{\infty}(\Omega)_{+}, C>$ 0 , and $1 \leq r<p^{*}$;

(ii) If $F(x, t)=\int_{0}^{t} f(x, s) d s$, there exists $\theta \in L^{\infty}(\Omega), \theta \leq 0, \theta \neq 0$, such that

$$
\limsup _{|s| \rightarrow \infty} \frac{p F(x, s)}{|s|^{p}} \leq \theta(x) \quad \text { uniformly for a.a. } x \in \Omega ;
$$

(iii) There exist $\delta_{0}>0$ and $\eta>\lambda_{1}$ such that (with $C_{1}$ as in $(H)$ )

$$
\frac{C_{1} \eta}{p(p-1)}|s|^{p} \leq F(x, s) \quad \text { for a.a. } x \in \Omega \text {, all }|s| \leq \delta_{0} ;
$$

(iv) There exists $\lambda>0$ such that

$$
\left(f(x, s)+\lambda|s|^{p-2} s\right) s \geq 0 \text { for a.a. } x \in \Omega \text {, all } s \in \mathbb{R} .
$$

Example 5.1 A simple example of function satisfying assumption $\left(H_{f}\right)_{1}$ is the following one:

$$
f(s)= \begin{cases}\hat{\eta}|s|^{p-2} s & \text { if }|s| \leq 1 \\ C|s|^{q-2} s-(C-\hat{\eta})|s|^{p-2} s & \text { if }|s|>1\end{cases}
$$

where $\hat{\eta}>\frac{C_{1} \lambda_{1}}{p-1}, C>\hat{\eta}$, and $1<q<p$.

The subsequent result on multiple solutions to problem (2) can be found in [22, Theorem 4.2]. In the statement of it we use the space

$$
C_{n}^{1}(\bar{\Omega})=\left\{u \in C^{1}(\bar{\Omega}): \frac{\partial u}{\partial n}=0 \text { on } \partial \Omega\right\} .
$$


The Banach space $C^{1}(\bar{\Omega})$ is an ordered Banach space with the positive cone

$$
C_{+}=\left\{u \in C^{1}(\bar{\Omega}): u(x) \geq 0 \text { for all } x \in \bar{\Omega}\right\} .
$$

This cone has a nonempty interior which is given by

$$
\operatorname{int} C_{+}=\left\{u \in C_{+}: u(x)>0 \text { for all } x \in \bar{\Omega}\right\} .
$$

Theorem 5.1 Let the hypotheses $(H)$ and $\left(H_{f}\right)_{1}$ be satisfied. Then problem (2) possesses at least three nontrivial smooth solutions $u_{0} \in \operatorname{int} C_{+}, v_{0} \in-\operatorname{int} C_{+}$and $y_{0} \in C_{n}^{1}(\bar{\Omega})$.

\section{Proof (sketch)}

For $\lambda>0$ as in $\left(H_{f}\right)_{1}(\mathrm{iv})$, we introduce the function

$$
f_{+}^{\lambda}(x, s)= \begin{cases}0 & \text { if } s \leq 0 \\ f(x, s)+\lambda s^{p-1} & \text { if } s>0 .\end{cases}
$$

Let $F_{+}^{\lambda}(x, t)=\int_{0}^{t} f_{+}^{\lambda}(x, s) d s$. Consider the $C^{1}$-functional $\varphi_{+}^{\lambda}: W^{1, p}(\Omega) \rightarrow \mathbb{R}$ defined by

$$
\varphi_{+}^{\lambda}(u)=\int_{\Omega} G(x, \nabla u) d x+\frac{\lambda}{p}\|u\|_{p}^{p}-\int_{\Omega} F_{+}^{\lambda}(x, u) d x \text { for all } u \in W^{1, p}(\Omega) .
$$

By hypotheses $\left(H_{f}\right)_{1}(\mathrm{i})$, (ii) and Proposition $2.1, \varphi_{+}^{\lambda}$ is coercive and so we can find $u_{0} \in W^{1, p}(\Omega)$ such that

$$
\varphi_{+}^{\lambda}\left(u_{0}\right)=\inf _{W^{1, p}(\Omega)} \varphi_{+}^{\lambda} .
$$

From $\left(H_{f}\right)_{1}$ (iii) we have that $\varphi_{+}^{\lambda}\left(u_{0}\right)<0$, hence $u_{0} \neq 0$. Also we note that

$$
V\left(u_{0}\right)+\lambda\left|u_{0}\right|^{p-2} u_{0}=f_{+}^{\lambda}\left(x, u_{0}\right) .
$$

Therefore $u_{0}$ is a nontrivial solution of problem (2) with $u \in L^{\infty}(\Omega)$ (see [33]).

On account of the nonlinear regularity theory (see [17]), we infer that $u_{0} \in C_{+} \backslash\{0\}$, while hypothesis $\left(H_{f}\right)_{1}$ (iv) implies

$$
-\operatorname{div} A\left(x, \nabla u_{0}(x)\right)+\lambda u_{0}(x)^{p-1}=f\left(x, u_{0}(x)\right)+\lambda u_{0}(x)^{p-1} \geq 0 \text { a.e. in } \Omega .
$$

Invoking the nonlinear maximum principle in [20, Theorem A], we conclude that $u_{0} \in \operatorname{int} C_{+}$. Similarly, we get $v_{0} \in-\operatorname{int} C_{+}$.

Recall the $C^{1}$-energy functional $\varphi: W^{1, p}(\Omega) \rightarrow \mathbb{R}$ associated with problem (2) defined in (6) and note that $\left.\varphi\right|_{C_{+}}=\left.\varphi_{+}^{\lambda}\right|_{C_{+}}$. So $u_{0} \in \operatorname{int} C_{+}$is a local $C^{1}(\bar{\Omega})$-minimizer of $\varphi$. On the basis of Theorem 3.1, we infer that $u_{0}$ is a local $W^{1, p}(\Omega)$-minimizer of $\varphi$. Similarly, we have that $v_{0} \in-\operatorname{int} C_{+}$is a local $W^{1, p}(\Omega)$-minimizer of $\varphi$. 
Now we will provide a third nontrivial solution of problem (2). Without any loss of generality, we may assume that $\varphi$ has a finite critical set. Then we can find $\rho>0$ such that

$$
\varphi\left(v_{0}\right) \leq \varphi\left(u_{0}\right)<\inf \left[\varphi(u):\left\|u-u_{0}\right\|=\rho\right]=: m_{\rho} .
$$

Moreover, we have that $\varphi$ is coercive; hence it satisfies the Palais-Smale condition. Consequently, the mountain pass theorem can be applied giving $y_{0} \in W^{1, p}(\Omega)$ such that $\varphi\left(y_{0}\right) \geq m_{\rho}$ and $\varphi^{\prime}\left(y_{0}\right)=0$. As before, through the nonlinear regularity theory, it follows that $y_{0} \in C_{n}^{1}(\bar{\Omega})$ is a solution of problem (2). By (9), it turns out that $y_{0} \notin\left\{u_{0}, v_{0}\right\}$.

It remains to show that $y_{0} \neq 0$. From the mountain pass theorem we have the minimax characterization

$$
\varphi\left(y_{0}\right)=\inf _{\gamma \in \Gamma} \max _{t \in[0,1]} \varphi(\gamma(t)),
$$

where

$$
\Gamma=\left\{\gamma \in C\left([0,1], W^{1, p}(\Omega)\right): \gamma(0)=v_{0}, \gamma(1)=u_{0}\right\}
$$

If we can produce a path $\gamma_{0} \in \Gamma$ such that $\left.\varphi\right|_{\gamma_{0}}<0$, then $\varphi\left(y_{0}\right)<0=\varphi(0)$ and so $y_{0} \neq 0$ and we are done.

In what follows we generate such a path $\gamma_{0} \in \Gamma$. Consider the sets $M=$ $W^{1, p}(\Omega) \cap \partial B_{1}^{L^{p}}$ endowed with the relative $W^{1, p}(\Omega)$-topology and $M_{c}=M \cap C^{1}(\bar{\Omega})$ furnished with the relative $C^{1}(\bar{\Omega})$-topology. We notice that $M_{c}$ is dense in $M$. So, if we set

$$
\hat{\Gamma}=\left\{\hat{\gamma} \in C([-1,1], M): \hat{\gamma}(-1)=-\hat{u}_{0}, \hat{\gamma}(1)=\hat{u}_{0}\right\}
$$

and

$$
\hat{\Gamma}_{c}=\left\{\hat{\gamma} \in C\left([-1,1], M_{c}\right): \hat{\gamma}(-1)=-\hat{u}_{0}, \hat{\gamma}(1)=\hat{u}_{0}\right\}
$$

we have that $\hat{\Gamma}_{c}$ is dense in $\hat{\Gamma}$. Then, from [1], given $\varepsilon>0$ we can find $\hat{\gamma}_{0} \in \hat{\Gamma}_{c}$ with

$$
\max \left[\|\nabla u\|_{p}^{p}: u \in \hat{\gamma}_{0}([-1,1])\right] \leq \lambda_{1}+\varepsilon .
$$

Here $\lambda_{1}$ stands for the second eigenvalue (the first nontrivial eigenvalue) of $-\Delta_{p}$ on $W^{1, p}(\Omega)$. The corresponding result for the Dirichlet problem can be found in [7]. For $\eta>\lambda_{1}$ in $\left(H_{f}\right)_{1}$ (iii), let $\varepsilon>0$ with $\lambda_{1}+\varepsilon<\eta$. Also let $\xi>0$ be such that $\xi|u(x)| \leq \delta_{0}$ for all $x \in \bar{\Omega}$, all $u \in \hat{\gamma}_{0}([-1,1])$, with $\delta_{0}>0$ in $\left(H_{f}\right)_{1}$ (iii). Then by $\left(H_{f}\right)_{1}$ (iii) we deduce that $\varphi(\xi u)<0$ for all $u \in \hat{\gamma}_{0}([-1,1])$. It follows that $\bar{\gamma}_{0}=\xi \hat{\gamma}_{0}$ is a path from $-\xi \hat{u}_{0}$ to $\xi \hat{u}_{0}$ with $\left.\varphi\right|_{\bar{\gamma}_{0}}<0$.

Without loss of generality we may assume that $\left\{0, u_{0}\right\}$ are the only critical points of $\varphi_{+}^{\lambda}$ (if $y \in W^{1, p}(\Omega)$ is another critical point of $\varphi_{+}^{\lambda}$, then $y \in \operatorname{int} C_{+}$, thus it is a third nontrivial smooth solution). Set $a:=\varphi_{+}^{\lambda}\left(u_{0}\right)=\varphi\left(u_{0}\right)<0=\varphi_{+}^{\lambda}(0)$. Hence we can apply the second deformation theorem (see, e.g., [11, p. 628]) and 
obtain a homotopy $h:[0,1] \times\left(\left(\varphi_{+}^{\lambda}\right)^{0} \backslash K_{\varphi_{+}^{\lambda}}^{0}\right) \rightarrow\left(\varphi_{+}^{\lambda}\right)^{0}$ satisfying the properties stated in that theorem, where $\left(\varphi_{+}^{\lambda}\right)^{0}=\left(\varphi_{+}^{\lambda}\right)^{-1}(-\infty, 0]$ and $K_{\varphi_{+}^{\lambda}}^{0}$ denotes the set of critical points at the level 0 . Let $\gamma_{+}(t)=\left(h\left(t, \xi \hat{u}_{0}\right)\right)^{+}$for all $t \in[0,1]$. Then $\gamma_{+}(0)=\xi \hat{u}_{0} \quad\left(\hat{u}_{0}\right.$ is the $L^{p}$-normalized eigenfunction of the negative Neumann $p$-Laplacian corresponding to the eigenvalue 0 , that is, $\hat{u}_{0}(x)=1 /|\Omega|_{N}^{1 / p}$ ) and $\gamma_{+}(1)=u_{0}$ because $\left(\varphi_{+}^{\lambda}\right)^{a}=\left(\varphi_{+}^{\lambda}\right)^{-1}(-\infty, a]=\left\{u_{0}\right\}$. Consequently, we get

$$
\left.\varphi\right|_{\gamma_{+}}<0 \text {. }
$$

In a similar fashion we produce a path $\gamma_{-}$joining $v_{0}$ and $-\xi \hat{u}_{0}$ such that

$$
\left.\varphi\right|_{\gamma_{-}}<0 \text {. }
$$

Finally, we concatenate $\gamma_{-}, \bar{\gamma}_{0}$ and $\gamma_{+}$to construct a path $\gamma_{0} \in \Gamma$ satisfying $\left.\varphi\right|_{\gamma_{0}}<0$, which completes the proof.

\section{Positive Solutions}

In this section we seek multiple positive solutions for the special case of problem (1) where in place of the general operator $\operatorname{div} A(x, \nabla u)$ we take the $p$-Laplacian $\Delta_{p}$ with $1<p<+\infty$.

Let $\Omega \subset \mathbb{R}^{N}$ be a bounded domain with a $C^{2}$-boundary $\partial \Omega$. Given $\beta \in L^{\infty}(\Omega) \backslash$ $\{0\}, \beta \geq 0$, we consider for $1<q<p$ the nonlinear Dirichlet problem:

$$
\begin{cases}-\Delta_{p} u(x)=\beta(x)|u(x)|^{q-2} u(x)+f(x, u(x)) & \text { in } \Omega, \\ u=0 & \text { on } \partial \Omega,\end{cases}
$$

where $\Delta_{p}$ denotes the $p$-Laplace differential operator defined on $W_{0}^{1, p}(\Omega)$ by

$$
\Delta_{p} u=\operatorname{div}\left(|\nabla u|^{p-2} \nabla u\right) .
$$

Note that in (10), the right-hand side nonlinearity contains a $(p-1)$-sublinear term (usually, called "concave term") $\beta(x)|u(x)|^{q-2} u(x)$, with $1<q<p$, and an additional Carathéodory term $f(x, u)$.

Denote by $\lambda_{1}$ the first eigenvalue of the negative Dirichlet $p$-Laplacian operator $\left(-\Delta_{p}, W_{0}^{1, p}(\Omega)\right)$ and by $\hat{u}_{1}$ the $L^{p}$-normalized positive eigenfunction corresponding to $\lambda_{1}$. Through the nonlinear regularity theory (see [17]) and the nonlinear strong maximum principle of Vazquez [30], we have that $\hat{u}_{1} \in \operatorname{int} C_{+}$, where this time we denote

$$
C_{+}=\left\{u \in C^{1}(\bar{\Omega}): u(x) \geq 0 \text { for all } x \in \bar{\Omega} \text { and } u(x)=0 \text { on } \partial \Omega\right\} .
$$

This cone has a nonempty interior equal to

$$
\operatorname{int} C_{+}=\left\{u \in C_{+}: u(x)>0 \text { for all } x \in \Omega \text { and } \frac{\partial u}{\partial n}(x)<0 \text { for all } x \in \partial \Omega\right\} \text {. }
$$


As before, $n$ stands for the outward unit normal on $\partial \Omega$.

The hypotheses for the nonlinearity $f(x, u)$ in $(10)$ are as follows:

$\left(H_{f}\right)_{2} f: \Omega \times \mathbb{R} \rightarrow \mathbb{R}$ is a function such that

(i) For all $s \in \mathbb{R}, x \mapsto f(x, s)$ is measurable;

(ii) For a.a. $x \in \Omega, x \mapsto f(x, s)$ is continuous and $f(x, 0)=0$;

(iii) For a.a. $x \in \Omega$ and all $s \in \mathbb{R}$, we have $|f(x, s)| \leq a(x)+c|s|^{r-1}$, with $a \in L^{\infty}(\Omega)_{+}, c>0$ and $p<r<p^{*}$;

(iv) If $F(x, s)=\int_{0}^{s} f(x, t) d t$, then

(iv.a) $\lim _{s \rightarrow+\infty} \frac{F(x, s)}{s^{p}}=+\infty$ uniformly for a.a. $x \in \Omega$,

(iv.b) there exist $\tau \in\left(\max \left\{\frac{r-p}{p} N, r-p\right\}, p^{*}\right)$ and $\gamma_{0}>0$, such that $\tau>q$ and

$$
\liminf _{s \rightarrow+\infty} \frac{f(x, s) s-p F(x, s)}{s^{\tau}}>\gamma_{0} \text { uniformly for a.a. } x \in \Omega
$$

(v) There exist $\xi, \theta \in L^{\infty}(\Omega)_{+}$, such that $\theta(x) \leq \lambda_{1}$ a.e. on $\Omega$, with strict inequality on a set of positive measure, and

$$
-\xi(x) \leq \liminf _{s \rightarrow 0^{+}} \frac{f(x, s)}{s^{p-1}} \leq \limsup _{s \rightarrow 0^{+}} \frac{f(x, s)}{s^{p-1}} \leq \theta(x) \text { uniformly for a.a. } x \in \Omega .
$$

The following theorem can be found in [28].

Theorem 6.1 If hypotheses $\left(H_{f}\right)_{2}$ hold, then there exists $\xi^{*}>0$ such that if $\|\beta\|_{\infty}<\xi^{*}$, then problem (10) has at least two distinct (nontrivial) positive solutions $u_{0}, \hat{u} \in \operatorname{int} C_{+}$.

Proof (sketch)

We introduce the following truncation of the right-hand side of the equation in (10):

$$
\hat{f}_{+}(x, s)= \begin{cases}0 & \text { if } s \leq 0 \\ \beta(x) s^{q-1}+f(x, s) & \text { if } s>0\end{cases}
$$

which is a Carathéodory function.

Next we set $\hat{F}_{+}(x, s)=\int_{0}^{s} \hat{f}_{+}(x, t) d t$ and then consider the functional $\hat{\varphi}_{+} \in$ $C^{1}\left(W_{0}^{1, p}(\Omega)\right)$ defined by

$$
\hat{\varphi}_{+}(u)=\frac{1}{p}\|\nabla u\|_{p}^{p}-\int_{\Omega} \hat{F}_{+}(x, u(x)) d x \text { for all } u \in W_{0}^{1, p}(\Omega) .
$$

From hypotheses $\left(H_{f}\right)_{2}$ (i)-(iv) we derive that $\hat{\varphi}_{+}$satisfies the Cerami condition. Then the first positive solution $u_{0}$ is obtained through the mountain pass theorem, which leads to

$$
\hat{\varphi}_{+}\left(u_{0}\right) \geq \inf _{\partial B_{\rho}} \hat{\varphi}_{+}=: \hat{\eta}_{+}>0
$$

where $\partial B_{\rho}$ denotes the sphere in $W_{0}^{1, p}(\Omega)$ centered at 0 and of a small radius $\rho>0$. 
Now we generate a second positive solution for problem (10). By $\left(H_{f}\right)_{2}(\mathrm{v})$, we find constants $\hat{c}_{0}>0$ and $\delta>0$ such that

$$
F(x, s) \geq-\frac{\hat{c}_{0}}{p} s^{p} \text { for a.a. } x \in \Omega, \text { all } s \in[0, \delta] .
$$

Let $v \in C_{+}$with $v>0$ in $\Omega$ and $\|v\|=\|\nabla v\|_{p}=1$. For $t>0$ small such that $t v(x) \in[0, \delta]$ for all $x \in \bar{\Omega}$, we have the estimate

$$
\hat{\varphi}_{+}(t v) \leq t^{q}\left[\frac{t^{p-q}}{p}\left(1+\frac{\hat{c}_{0}}{\lambda_{1}}\right)-\frac{1}{q} \int_{\Omega} \beta(x) v(x)^{q} d x\right]
$$

since $1=\|\nabla v\|_{p}^{p} \geq \lambda_{1}\|v\|_{p}^{p}$. Choosing $t>0$ possibly smaller and recalling that $p>q$, we have

$$
\hat{\varphi}_{+}(t v)<0 \text { and } t\|v\|=t \leq \rho .
$$

By (13) we have that

$$
\eta:=\hat{\eta}_{+}-\inf _{\bar{B}_{\rho}} \hat{\varphi}_{+}>0
$$

where $\bar{B}_{\rho}=\left\{u \in W_{0}^{1, p}(\Omega):\|u\| \leq \rho\right\}$.

Let $\varepsilon \in(0, \eta)$. By the Ekeland's variational principle, there exists $u_{\varepsilon} \in \bar{B}_{\rho}$ such that

$$
\hat{\varphi}_{+}\left(u_{\varepsilon}\right) \leq \inf _{\bar{B}_{\rho}} \hat{\varphi}_{+}+\varepsilon
$$

and

$$
\hat{\varphi}_{+}\left(u_{\varepsilon}\right) \leq \hat{\varphi}_{+}(y)+\varepsilon\left\|y-u_{\varepsilon}\right\| \text { for all } y \in \bar{B}_{\rho} .
$$

From inequality (14) and since $\varepsilon<\eta$, we obtain that $\hat{\varphi}_{+}\left(u_{\varepsilon}\right)<\inf _{\partial B_{\rho}} \hat{\varphi}_{+}$. It follows that $u_{\varepsilon} \in B_{\rho}=\left\{u \in W_{0}^{1, p}(\Omega):\|u\|<\rho\right\}$, so for every $h \in W_{0}^{1, p}(\Omega)$ one has $u_{\varepsilon}+t h \in B_{\rho}$ whenever $t>0$ is sufficiently small. Inserting $y=u_{\varepsilon}+t h$ in (15), dividing by $t$ and then letting $t \rightarrow 0$ lead to $\left\|\hat{\varphi}_{+}^{\prime}\left(u_{\varepsilon}\right)\right\| \leq \varepsilon$.

Fix now a sequence $\varepsilon_{n} \downarrow 0$ and denote for simplicity $u_{n}=u_{\varepsilon_{n}}$. Then one has $\hat{\varphi}_{+}^{\prime}\left(u_{n}\right) \rightarrow 0$ and also $\left(1+\left\|u_{n}\right\|\right) \hat{\varphi}_{+}^{\prime}\left(u_{n}\right) \rightarrow 0$ as $n \rightarrow \infty$. Through the Cerami condition, this guarantees the existence of $\hat{u} \in W_{0}^{1, p}(\Omega)$ such that, along a relabeled subsequence, $u_{n} \rightarrow \hat{u}$ in $W_{0}^{1, p}(\Omega)$, which enables us to pass to limit in (14) to obtain

$$
\hat{\varphi}_{+}(\hat{u})=\inf _{\bar{B}_{\rho}} \hat{\varphi}_{+}<0=\hat{\varphi}_{+}(0)
$$

thereby $\hat{u} \neq 0$. Moreover, from (12) we see that $\hat{u} \neq u_{0}$. Since $\hat{\varphi}_{+}^{\prime}\left(u_{n}\right) \rightarrow 0$ and $u_{n} \rightarrow \hat{u}$, we infer that $\hat{\varphi}_{+}^{\prime}(\hat{u})=0$. Then, as for $u_{0}$, we obtain that $\hat{u}$ solves problem (10) and $\hat{u} \in \operatorname{int} C_{+}$. 
Remark 1 If the right-hand side of the equation in problem (10) has the special form

$$
\lambda|u|^{q-2} u+\left(u^{+}\right)^{\tau-1} \text { with } \lambda>0 \text { and } q<p<\tau<p^{*},
$$

Theorem 6.1 recovers the existence result in the case of $p$-Laplacian with $1<p<$ $+\infty$ due to García Azorero et al. [10] and the analogous result of Guo and Zhang [14] for $p \geq 2$.

\section{Extremal Solutions}

Consider the particular form of problem (10) restricting $\beta$ to constants $\lambda>0$, that is

$$
\begin{cases}-\Delta_{p} u(x)=\lambda|u(x)|^{q-2} u(x)+f(x, u(x)) & \text { in } \Omega, \\ u=0 & \text { on } \partial \Omega .\end{cases}
$$

We recall that here we have, as in Sect. 6, $1<q<p$. We slightly modify our assumptions:

$\left(H_{f}\right)_{3} \quad f: \Omega \times \mathbb{R} \rightarrow \mathbb{R}$ satisfies hypotheses $\left(H_{f}\right)_{2}(\mathrm{i})-(\mathrm{iv})$, and in addition

(vi) $\lim _{s \rightarrow 0^{+}} \frac{f(x, s)}{s^{p-1}}=0$ uniformly for a.a. $x \in \Omega$;

(vii) (local sign condition) there is a number $b>0$ such that

$$
f(x, s) \geq 0 \text { for a.a. } x \in \Omega \text { and all } s \in(0, b) .
$$

The aim of this section is to study the extremal solutions for problem (16), which means the biggest negative solution and the smallest positive solution of (16). To this end we recall that $\lambda_{1}$ denotes the first eigenvalue of the negative Dirichlet $p$-Laplacian $\left(-\Delta_{p}, W_{0}^{1, p}(\Omega)\right)$ and $\hat{u}_{1}$ stands for the $L^{p}$-normalized positive eigenfunction corresponding to $\lambda_{1}$. There holds $\hat{u}_{1} \in \operatorname{int} C_{+}$, with $C_{+}$given in (11). The result below can be found in [28].

Proposition 7.1 If hypotheses $\left(H_{f}\right)_{3}$ hold, then there is $\lambda^{*}>0$ such that for all $\lambda \in\left(0, \lambda^{*}\right)$, problem (16) has a smallest positive solution $u_{0} \in \operatorname{int} C_{+}$satisfying $\left\|u_{0}\right\|_{\infty}<b$.

Proof

First, we look for suitable positive sub-supersolutions of problem (16). Hypothesis $\left(H_{f}\right)_{3}(\mathrm{vi})$ implies that for any $\varepsilon>0$, we can find $\hat{c}_{\varepsilon}>0$ such that

$$
f(x, s) \leq \varepsilon s^{p-1}+\hat{c}_{\varepsilon} s^{r-1} \text { for a.a. } x \in \Omega \text { and all } s>0 .
$$

Let $e \in \operatorname{int} C_{+}$solve

$$
\begin{cases}-\Delta_{p} e(x)=1 & \text { in } \Omega \\ e=0 & \text { on } \partial \Omega\end{cases}
$$


Notice that there exists $\lambda^{*}>0$ such that for all $\lambda \in\left(0, \lambda^{*}\right)$ there is $\eta_{1}=\eta_{1}(\lambda) \in$ $\left(0, b /\|e\|_{\infty}\right)$ with

$$
\lambda\left(\eta_{1}\|e\|_{\infty}\right)^{q-1}+\varepsilon\left(\eta_{1}\|e\|_{\infty}\right)^{p-1}+\hat{c}_{\varepsilon}\left(\eta_{1}\|e\|_{\infty}\right)^{r-1}<\eta_{1}^{p-1} .
$$

Fix $\lambda \in\left(0, \lambda^{*}\right)$ and set $\bar{u}=\eta_{1} e$. Then we see that

$$
\begin{aligned}
-\Delta_{p} \bar{u}=\eta_{1}^{p-1}>\lambda\left(\eta_{1}\|e\|_{\infty}\right)^{q-1}+\varepsilon\left(\eta_{1}\|e\|_{\infty}\right)^{p-1} & +\hat{c}_{\varepsilon}\left(\eta_{1}\|e\|_{\infty}\right)^{r-1} \\
& \geq \lambda \bar{u}^{q-1}+f(z, \bar{u}) .
\end{aligned}
$$

This expresses that $\bar{u} \in \operatorname{int} C_{+}$is a (strict) supersolution for problem (16). Moreover, we have $\|\bar{u}\|_{\infty}<b$.

Choose $\tilde{\varepsilon}>0$ so small that $\tilde{\varepsilon} \hat{u}_{1}(x)<\bar{u}(x)$ for all $x \in \Omega$ and $\lambda_{1} \tilde{\varepsilon}^{p-q} \hat{u}_{1}^{p-q}<\lambda$. Set $\underline{u}=\tilde{\varepsilon} \hat{u}_{1}$. By hypothesis $\left(H_{f}\right)_{3}$ (vii) we infer that

$$
-\Delta_{p} \underline{u}=\lambda_{1} \tilde{\varepsilon}^{p-1} \hat{u}_{1}^{p-1}<\lambda \tilde{\varepsilon}^{q-1} \hat{u}_{1}^{q-1} \leq \lambda \underline{u}^{q-1}+f(x, \underline{u}) .
$$

It follows that $\underline{u} \in \operatorname{int} C_{+}$is a (strict) subsolution for problem (16), and in addition we have $\underline{u} \leq \bar{u}$.

Consider now the sequence $\underline{u}_{n}=\tilde{\varepsilon}_{n} \hat{u}_{1}$ with $\tilde{\varepsilon}_{n} \downarrow 0$. Since the problem (16) has a smallest solution $u_{*}^{n}$ in the ordered interval $\left[\underline{u}_{n}, \bar{u}\right]$ and it satisfies $u_{*}^{n} \in \operatorname{int} C_{+}$, it follows that the sequence $\left\{u_{*}^{n}\right\}_{n \geq 1} \subset W_{0}^{1, p}(\Omega)$ is bounded. Acting with $u_{*}^{n}-u_{+} \in$ $W_{0}^{1, p}(\Omega)$ on

$$
-\Delta_{p} u_{*}^{n}=\lambda\left(u_{*}^{n}\right)^{q-1}+f\left(\cdot, u_{*}^{n}(\cdot)\right)
$$

results in

$$
\lim _{n \rightarrow \infty}\left\langle-\Delta_{p} u_{*}^{n}, u_{*}^{n}-u_{+}\right\rangle=0 .
$$

The $(\mathrm{S})_{+}$property for the $p$-Laplacian (see also Proposition 2.2) enables us to get $u_{*}^{n} \rightarrow u_{+}$in $W_{0}^{1, p}(\Omega)$ as $n \rightarrow \infty$. It follows that $u_{+}$is a solution of the problem (16). The nonlinear regularity theory and strong maximum principle imply that $u_{+} \in \operatorname{int} C_{+}$ provided $u_{+} \neq 0$.

Let us prove that $u_{+} \neq 0$. To this end we introduce the function $\psi: W_{0}^{1, p}(\Omega) \rightarrow \mathbb{R}$ by

$$
\psi(u)=\frac{1}{p}\|\nabla u\|_{p}^{p}-\frac{\lambda}{q}\left\|u^{+}\right\|_{q}^{q} \text { for all } u \in W_{0}^{1, p}(\Omega) .
$$

Here $u^{+}$denotes the positive part of the function $u$. Since $\psi$ coercive and sequentially weakly lower semicontinuous, and taking into account that $q<p$, there is $u \in W_{0}^{1, p}(\Omega)$ such that

$$
\psi(u)=\inf _{W_{0}^{1, p}(\Omega)} \psi<0
$$

so $u \neq 0$ and

$$
-\Delta_{p} u-\lambda\left(u^{+}\right)^{q-1}=0 .
$$


Acting with $-u^{-} \in W_{0}^{1, p}(\Omega)$, where $u^{-}$denotes the negative part of the function $u$, ensures that $u \geq 0$, thus $u$ solves the problem

$$
\begin{cases}-\Delta_{p} u(x)=\lambda u(x)^{q-1} & \text { in } \Omega \\ u=0 & \text { on } \partial \Omega .\end{cases}
$$

This renders $\Delta_{p} u(x) \leq 0$ a.e. in $\Omega$, which entails $u \in \operatorname{int} C_{+}$due to the strong maximum principle [30].

Since $u_{*}^{n} \in \operatorname{int} C_{+}$, there is the biggest $\xi_{n}>0$ such that $\xi_{n} u \leq u_{*}^{n}$ for all $n \geq 1$. If $\xi_{n}<1$, then we have

$$
-\Delta_{p} u_{*}^{n}(x) \geq \lambda u_{*}^{n}(x)^{q-1} \geq \lambda \xi_{n}^{q-1} u(x)^{q-1}>\lambda \xi_{n}^{p-1} u(x)^{q-1}=-\Delta_{p}\left(\xi_{n} u\right)(x) .
$$

By means of the comparison principle in [13], we arrive at $u_{*}^{n}-\xi_{n} u \in \operatorname{int} C_{+}$, which contradicts the maximality of $\xi_{n}$.

Therefore $\xi_{n} \geq 1$, which implies that $u \leq u_{*}^{n}$. We conclude that $u \leq u_{+}$, so $u_{+} \neq 0$. Now it is straightforward to check that $u_{+}$is indeed the smallest positive solution of (16). The proof is thus complete.

Next we deal with the biggest negative solution. We work under asymmetrical situations on $\mathbb{R}_{+}$and $\mathbb{R}_{-}$. We impose the hypotheses:

$\left(H_{f}\right)_{4} f: \Omega \times \mathbb{R} \rightarrow \mathbb{R}$ satisfies hypotheses $\left(H_{f}\right)_{2}$ (i)-(iii) and in addition

(viii) There exists $\theta \in L^{\infty}(\Omega)_{+}$such that $\theta(x) \leq \lambda_{1}$ a.e. on $\Omega$, with strict inequality on a set of positive measure, and

$$
\limsup _{s \rightarrow-\infty} \frac{f(x, s)}{|s|^{p-2} s} \leq \theta(x) \text { uniformly for a.a. } x \in \Omega
$$

(ix)

$$
\lim _{s \rightarrow 0^{-}} \frac{f(x, s)}{|s|^{p-2} s}=0 \text { uniformly for a.a. } x \in \Omega
$$

(x) (local sign condition) There is a number $b>0$ such that $f(x, s) \leq 0$ for a.a. $x \in \Omega$ and all $s \in(-b, 0)$.

Remark 2 Assumption $\left(H_{f}\right)_{4}$ (viii) guarantees that for a.a. $x \in \Omega, f(x, \cdot)$ has a $(p-1)$-(sub)linear growth near $-\infty$.

We have the following result from [28].

Proposition 7.2 If hypotheses $\left(H_{f}\right)_{4}$ hold, then for every $\lambda>0$, problem (16) has a biggest negative solution $v_{-} \in-\operatorname{int} C_{+}$.

Proof (sketch)

First we search for negative sub-supersolutions of problem (16). By $\left(\mathrm{H}_{f}\right)_{2}(\mathrm{iii})$, $\left(\mathrm{H}_{f}\right)_{4}\left(\right.$ viii) we have that given $\varepsilon>0$ we can find $\xi_{\varepsilon} \in L^{\infty}(\Omega)_{+} \backslash\{0\}$ such that

$$
f(x, s) \geq(\theta(x)+\varepsilon)|s|^{p-2} s-\xi_{\varepsilon}(x) \text { for a.a. } x \in \Omega \text { and all } s \leq 0 .
$$

Define the Carathéodory function

$$
g_{\lambda}(x, s)= \begin{cases}\lambda|s|^{q-2} s+(\theta(x)+\varepsilon)|s|^{p-2} s-\xi_{\varepsilon}(x) & \text { if } s \leq 0 \\ -\xi_{\varepsilon}(x) & \text { if } s>0\end{cases}
$$


and consider the $C^{1}$-functional $\sigma: W_{0}^{1, p}(\Omega) \rightarrow \mathbb{R}$ defined by

$$
\sigma(v)=\frac{1}{p}\|\nabla v\|_{p}^{p}-\int_{\Omega} G_{\lambda}(x, v(x)) d x \text { for all } v \in W_{0}^{1, p}(\Omega),
$$

where $G_{\lambda}(x, s)=\int_{0}^{s} g_{\lambda}(x, t) d t$. The function $\sigma$ is coercive and sequentially weakly lower semicontinuous, so there is $\underline{v} \in W_{0}^{1, p}(\Omega)$ such that

$$
\sigma(\underline{v})=\inf _{W_{0}^{1, p}(\Omega)} \sigma .
$$

We act with $\underline{v}^{+} \in W_{0}^{1, p}(\Omega)$ on the problem

$$
\begin{cases}-\Delta_{p} \underline{v}+\lambda\left(\underline{v}^{-}\right)^{q-1}+(\theta+\varepsilon)\left(\underline{v}^{-}\right)^{p-1}+\xi_{\varepsilon}=0 & \text { in } \Omega \\ \underline{v}=0 & \text { on } \partial \Omega,\end{cases}
$$

which implies that $\underline{v} \leq 0, \underline{v} \neq 0, \Delta_{p}(-\underline{v}) \leq 0$, so $\underline{v} \in-\operatorname{int} C_{+}$according to the strong maximum principle in [30]. Furthermore, $\underline{v}$ is a lower solution of problem (16). Given $\tilde{\varepsilon}>0$ small, set $\bar{v}=-\tilde{\varepsilon} \hat{u}_{1} \in-\operatorname{int} C_{+}$. Hypothesis $\left(H_{f}\right)_{4}$ (ix) ensures that $\bar{v}$ is an upper solution of (16) and $\underline{v} \leq \bar{v}$.

Consider the sequence of functions $\bar{v}_{n}=-\tilde{\varepsilon}_{n} \hat{u}_{1}$ corresponding to a sequence $\tilde{\varepsilon}_{n} \downarrow 0$. Then, for every integer $n \geq 1$, we get the biggest solution $v_{*}^{n} \in-\operatorname{int} C_{+}$ of problem (16) in the ordered interval $\left[\underline{v}, \bar{v}_{n}\right]$. Taking into account this maximality property and using once again the strong maximum principle (which is possible thanks to hypothesis $\left.\left(H_{f}\right)_{4}(\mathrm{x})\right)$, we establish that $v_{*}^{n} \rightarrow v_{-}$in $W_{0}^{1, p}(\Omega)$ for some $v_{-} \in-\operatorname{int} C_{+}$. Finally, we can show that $v_{-}$is the biggest negative solution of (16), which completes the proof.

\section{Nodal Solution}

Our main result on problem (16) asserts the existence of an additional solution which is nodal (sign changing). The complete proof of the theorem below as well as other related results can be found in [28].

Theorem 8.1 If hypotheses $\left(H_{f}\right)_{2}(i)-(i v),\left(H_{f}\right)_{3}(v i)-(v i i)$ and $\left(H_{f}\right)_{4}(v i i i)-(x)$ hold, then there exists $\lambda^{*}>0$ such that for all $\lambda \in\left(0, \lambda^{*}\right)$, problem (16) has at least four distinct nontrivial smooth solutions: $u_{0}, \hat{u} \in \operatorname{int} C_{+}$with $u_{0} \neq \hat{u}, v_{0} \in-\operatorname{int} C_{+}$ and $y_{0} \in C_{0}^{1}(\bar{\Omega}) \backslash\{0\}$ nodal.

\section{Proof (sketch)}

Theorem 6.1 and Proposition 7.2 provide a number $\lambda^{*}>0$ such that for every $\lambda \in\left(0, \lambda^{*}\right)$ there exist three constant sign solutions $u_{0}, \hat{u} \in \operatorname{int} C_{+}$and $v_{0} \in-\operatorname{int} C_{+}$ of problem (16). Fix any $\lambda \in\left(0, \lambda^{*}\right)$. In view of Propositions 7.1 and 7.2 there exist the smallest positive solution $u_{+} \in \operatorname{int} C_{+}$and the biggest negative solution $v_{-} \in-\mathrm{int} C_{+}$of problem (16). Consider the truncation:

$$
\hat{f}_{\lambda}(x, s)= \begin{cases}\lambda\left|v_{-}(x)\right|^{q-2} v_{-}(x)+f\left(x, v_{-}(x)\right) & \text { if } s<v_{-}(x) \\ \lambda|s|^{q-2} s+f(x, s) & \text { if } v_{-}(x) \leq s \leq u_{+}(x) \\ \lambda u_{+}(x)^{q-1}+f\left(x, u_{+}(x)\right) & \text { if } u_{+}(x)<s .\end{cases}
$$


Associated with this truncation we introduce the $C^{1}$ functional $\hat{\varphi}_{\lambda}: W_{0}^{1, p}(\Omega) \rightarrow \mathbb{R}$ defined by

$$
\hat{\varphi}_{\lambda}(u)=\frac{1}{p}\|\nabla u\|_{p}^{p}-\int_{\Omega} \hat{F}_{\lambda}(x, u(x)) d x \text { for all } u \in W_{0}^{1, p}(\Omega),
$$

where

$$
\hat{F}_{\lambda}(x, t)=\int_{0}^{t} \hat{f}_{\lambda}(x, s) d s \text { for all }(x, t) \in \Omega \times \mathbb{R} .
$$

Using the definition of the truncation function given above, it is straightforward to prove that every critical point of $\hat{\varphi}_{\lambda}$ is located in the ordered interval $\left[v_{-}, u_{+}\right]$. This can be checked by testing the equation $\hat{\varphi}_{\lambda}^{\prime}(u)=0$ with $\left(u-u_{+}\right)^{+}$and $\left(u-v_{-}\right)^{-}$. Then we can show that $v_{-}$and $u_{+}$are local minimizers of the functional $\hat{\varphi}_{\lambda}$. Moreover, we may assume that they are isolated critical points because otherwise we obtain infinitely many nodal solutions of problem (16).

Notice that the imposed assumptions permit to apply the mountain pass theorem to the functional $\hat{\varphi}_{\lambda}$. It follows that there exists a critical point $y_{0} \in\left[v_{-}, u_{+}\right]$of $\hat{\varphi}_{\lambda}$ with $y_{0} \neq v_{-}$and $y_{0} \neq u_{+}$. A major step in the proof is to establish that $y_{0} \neq 0$. In this respect we make use of the critical groups associated to the function $\hat{\varphi}_{\lambda}$ at $y_{0}$ and 0 . For the theory of critical groups we refer to [6] and [19].

The fact that $y_{0}$ is a critical point of $\hat{\varphi}_{\lambda}$ of mountain pass type implies that the critical group $C_{1}\left(\hat{\varphi}_{\lambda}, y_{0}\right)$ is nontrivial, that is $C_{1}\left(\hat{\varphi}_{\lambda}, y_{0}\right) \neq 0$. On the other hand, we can prove that for all $u \in W_{0}^{1, p}(\Omega)$ with $\|u\| \leq \rho$ and $\hat{\varphi}_{\lambda}(u) \leq 0$, we have $\hat{\varphi}_{\lambda}(t u) \leq 0$ whenever $t \in[0,1]$. In turn, this allows to develop a homotopy argument revealing that the critical groups $C_{k}\left(\hat{\varphi}_{\lambda}, 0\right)$ are all of them trivial, that is $C_{k}\left(\hat{\varphi}_{\lambda}, 0\right)=0$ for all $k$. Consequently, comparing the critical groups $C_{1}\left(\hat{\varphi}_{\lambda}, y_{0}\right)$ and $C_{1}\left(\hat{\varphi}_{\lambda}, 0\right)$, we derive that $y_{0} \neq 0$. Now we can conclude that the nontrivial solution $y_{0}$ of problem (16) is nodal. Indeed, relying on the location property $y_{0} \in\left[v_{-}, u_{+}\right]$, the solution $y_{0}$ must be sign changing because otherwise it is contradicted the maximality of $v_{-}$among the negative solutions or the minimality of $u_{+}$among the positive solutions. The proof is thus complete.

\section{New Problem}

In this section we consider the nonlinear Dirichlet problem

$$
\begin{cases}-\Delta_{p} u-\mu \Delta_{q} u=f(x, u, \nabla u) & \text { in } \Omega \\ u>0 & \text { in } \Omega \\ u=0 & \text { on } \partial \Omega\end{cases}
$$

on a bounded domain $\Omega$ in $\mathbb{R}^{N}$ with a $C^{1, \alpha}$-boundary $\partial \Omega$, for some $0<\alpha \leq 1$. In the left-hand side of the equation in $(N P)$ we have the $p$-Laplacian $\Delta_{p}$ and the 
$q$-Laplacian $\Delta_{q}$ with $1<q<p<+\infty$, and a constant $\mu \geq 0$. So, basically, the principal part of the elliptic equation is determined by the $(p, q)$-Laplacian operator $-\Delta_{p}-\Delta_{q}$. The right-hand side of the elliptic equation in problem $(N P)$ depends on the gradient $\nabla u$ of the solution $u$. This is a novelty with respect to problem (1). Problems where the right-hand side depends on the gradient of the solution are sometimes called with convection term.

We emphasize that the $(p, q)$-Laplacian operator is not homogeneous. However, it does not belong to the class of possibly nonhomogeneous operators described in statement $(H)$ (in the sense to be in the divergence form $\operatorname{div} A(x, \nabla(\cdot))$ with some $A(x, \cdot)$ satisfying $(H))$. Actually, all the conditions in $(H)$ are satisfied excepting $(H)$ (ii) that to fulfill our purpose should be replaced with

(ii') There exists a constant $C_{1}>0$ such that

$$
\left|D_{y} A(x, y)\right| \leq C_{1}\left(|y|^{p-2}+1\right) \quad \text { for every } x \in \bar{\Omega} \text { and } y \in \mathbb{R}^{N} \backslash\{0\} .
$$

If this is the case, then we can set $A(x, y)=|y|^{p}+\mu|y|^{q}$ for all $(x, y) \in \bar{\Omega} \times \mathbb{R}^{N}$ to comply with $(H)$.

We suppose that the nonlinearity $f(x, u, \nabla u)$ satisfies the hypothesis:

(FG) $f: \Omega \times \mathbb{R} \times \mathbb{R}^{N} \rightarrow \mathbb{R}$ is a Carathéodory function such that $f(x, 0, \xi)=0$ a.e. in $\Omega$, for all $\xi \in \mathbb{R}^{N}$, and

$$
b_{0}|t|^{r_{0}} \leq f(x, t, \xi) \leq b_{1}\left(1+|t|^{r_{1}}+|\xi|^{r_{2}}\right)
$$

for all $(x, t, \xi) \in \Omega \times \mathbb{R} \times \mathbb{R}^{N}$, with constants $b_{0}, b_{1}>0$, $r_{1}, r_{2} \in[0, p-1), r_{0} \in[0, p-1)$ if $\mu=0$, and $r_{0} \in[0, q-1)$ if $\mu>0$.

The following result can be found in [9].

Theorem 9.1 Under assumption ( $F G)$, problem $(N P)$ admits a (positive) solution $u \in C_{0}^{1}(\bar{\Omega})$.

Proof (sketch)

First, it is shown that for every $\varepsilon \in(0,1)$, the approximate problem

$$
\begin{cases}-\Delta_{p} u-\mu \Delta_{q} u=f(x, u+\varepsilon, \nabla u) & \text { in } \Omega \\ u>0 & \text { in } \Omega \\ u=0 & \text { on } \partial \Omega\end{cases}
$$

has at least a (positive) solution $u_{\varepsilon} \in C_{0}^{1}(\bar{\Omega})$. This conclusion is achieved through a Schauder basis of $W_{0}^{1, p}(\Omega)$ by using Brouwer's fixed point theorem on the corresponding finite dimensional spaces and then passing to the limit. For the solution $u_{\varepsilon} \in C_{0}^{1}(\bar{\Omega})$ of problem $\left(P_{\varepsilon}\right)$, from hypothesis $(F G)$ and

$$
\int_{\Omega}\left(\left|\nabla u_{\varepsilon}\right|^{p}+\mu\left|\nabla u_{\varepsilon}\right|^{q}\right) d x=\int_{\Omega} f\left(x, u_{\varepsilon}+\varepsilon, \nabla u_{\varepsilon}\right) u_{\varepsilon} d x,
$$


we get the estimate

$$
\left\|u_{\varepsilon}\right\|^{p} \leq C\left(\left\|u_{\varepsilon}\right\|+\left\|u_{\varepsilon}\right\|^{r_{1}+1}+\left\|u_{\varepsilon}\right\|^{r_{2}+1}\right),
$$

with a constant $C>0$ which is independent of $\varepsilon$. Since $1, r_{1}+1, r_{2}+1<p$, we infer that

$$
\left\|u_{\varepsilon}\right\| \leq C_{0},
$$

for a constant $C_{0}>0$ independent of $\varepsilon$. In view of (17), we can find $\varepsilon_{n} \rightarrow 0^{+}$such that the corresponding sequence $\left\{u_{n}=u_{\varepsilon_{n}}\right\}$ is strongly convergent

$$
u_{n} \rightarrow u \text { in } W_{0}^{1, p}(\Omega) \text { as } n \rightarrow \infty,
$$

with some $u \in W_{0}^{1, p}(\Omega)$. On the basis of (18) and because $u_{n}$ solves $\left(P_{\varepsilon_{n}}\right)$, it turns out that $u$ is a solution of the equation

$$
\begin{cases}-\Delta_{p} u-\mu \Delta_{q} u=f(x, u, \nabla u) & \text { in } \Omega \\ u=0 & \text { on } \partial \Omega .\end{cases}
$$

The regularity up to the boundary in [17, Theorem 1] and [18, p. 320] ensures that $u \in C^{1, \beta}(\bar{\Omega})$ with some $\beta \in(0,1)$. At this point we cannot guarantee that $u$ is nontrivial. In order to prove that $u>0$, we develop a comparison argument.

We note that there exists a (positive) solution $\underline{u} \in C_{0}^{1}(\bar{\Omega})$ to the problem

$$
\left\{\begin{array}{lll}
-\Delta_{p} u-\mu \Delta_{q} u=b_{0} u^{r_{0}} & \text { in } & \Omega \\
u>0 & \text { in } & \Omega \\
u=0 & \text { on } & \partial \Omega,
\end{array}\right.
$$

where $b_{0}$ and $r_{0}$ are the positive constants in hypothesis $(F G)$. In the sequel, $\underline{u}$ will be regarded as a subsolution of (19). We also observe that hypothesis $(F G)$ implies that $u_{\varepsilon}$ is a supersolution of problem (19) for each $\varepsilon \in(0,1)$.

Now we apply the comparison principle in [9] to problem (19), by taking the subsolution $\underline{u}$ and the supersolution $u_{\varepsilon}$. We emphasize that for applying this comparison principle it is essential to know that $u_{\varepsilon}>0$ in $\Omega$ as well as that $r_{0}<q-1$ if $\mu>0$, and $r_{0}<p-1$ if $\mu=0$. In order to apply the comparison principle we also need to check that

$$
\frac{u_{\varepsilon}}{\underline{u}}, \frac{\underline{u}}{u_{\varepsilon}} \in L^{\infty}(\Omega)
$$

To this end it suffices to show that whenever $x \rightarrow x_{0} \in \partial \Omega$ with $x \in \Omega$, one has

$$
\max \left\{\limsup _{x \rightarrow x_{0}} \frac{\underline{u}(x)}{u_{\varepsilon}(x)}, \limsup _{x \rightarrow x_{0}} \frac{u_{\varepsilon}(x)}{\underline{u}(x)}\right\}<+\infty .
$$

The property stated in (20) is established on the basis of the Hopf boundary point lemma in the strong maximum principle applied to both Dirichlet problems (19) and $\left(P_{\varepsilon}\right)$ with corresponding solutions $\underline{u}$ and $u_{\varepsilon}$, which amounts to saying that

$$
\frac{\partial \underline{u}}{\partial n}\left(x_{0}\right)<0, \frac{\partial u_{\varepsilon}}{\partial n}\left(x_{0}\right)<0 \text { for all } x_{0} \in \partial \Omega,
$$


where $n$ denotes the exterior normal unit vector on $\partial \Omega$. The Hopf boundary point lemma holds true for problems (19) and $\left(P_{\varepsilon}\right)$ by virtue of [29, Theorem 5.5.1]. Recalling that $u_{\varepsilon}, \underline{u} \in C^{1}(\bar{\Omega})$, it is clear from the L'Hôpital theorem and (21) that the property required in (20) is fulfilled. Therefore we can do the comparison of the solution $\underline{u}$ (regarded as a subsolution) of (19) with the supersolution $u_{\varepsilon}$ of (19) implying that

$$
u_{\varepsilon}(x) \geq \underline{u}(x)>0 \text { for all } x \in \Omega \text { and } \varepsilon \in(0,1) .
$$

Using (18), we can pass to the limit in (22) along a sequence $\varepsilon_{n} \rightarrow 0$. This leads to $u(x) \geq \underline{u}(x)>0$ for all $x \in \Omega$, so $u$ is a (positive) solution of problem $(N P)$, which completes the proof.

\section{References}

1. Aizicovici, S., Papageorgiou, N.S., Staicu, V.: The spectrum and an index formula for the Neumann p-Laplacian and multiple solutions for problems with a crossing nonlinearity. Discret. Contin. Dyn. Syst. 25(2), 431-456 (2009)

2. Barletta, G., Papageorgiou, N.S.: A multiplicity theorem for the Neumann $p$-Laplacian with an asymmetric nonsmooth potential. J. Glob. Optim. 39(3), 365-392 (2007)

3. Brezis, H., Nirenberg, L.: $H^{1}$ versus $C^{1}$ local minimizers. C. R. Acad. Sci. Paris Sér. I Math. 317(5), 465-472 (1993)

4. Carl, S., Perera, K.: Sign-changing and multiple solutions for the p-Laplacian. Abstr. Appl. Anal. 7(12), 613-625 (2002)

5. Carl, S., Le, V.K., Motreanu, D.: Nonsmooth Variational Problems and Their Inequalities. Comparison Principles and Applications. Springer Monographs in Mathematics. Springer, New York (2007)

6. Chang, K.-C.: Infinite-Dimensional Morse Theory and Multiple Solution Problems. Progress in Nonlinear Differential Equations and Their Applications, vol. 6. Birkhäuser, Boston, (1993)

7. Cuesta, M., de Figueiredo, D.G., Gossez, J.-P.: The beginning of the Fučik spectrum for the $p$-Laplacian. J. Differ. Equ. 159(1), 212-238 (1999)

8. Fan, X.: On the sub-supersolution method for $p(x)$-Laplacian equations. J. Math. Anal. Appl. 230(1), 665-682 (2007)

9. Faria, L.F.O., Miyagaki, O.H., Motreanu, D.: Comparison and positive solutions for problems with $(p, q)$-Laplacian and convection term. Proc. Edinburgh Math. Soc. (2014). doi:10.1017/S0013091513000576

10. García Azorero, J.P., Peral Alonso, I., Manfredi, J.J.: Sobolev versus Hölder local minimizers and global multiplicity for some quasilinear elliptic equations. Commun. Contemp. Math. 2(3), 385-404 (2000)

11. Gasinski, L., Papageorgiou, N.S.: Nonlinear Analysis. Chapman Hall/CRC Press, Boca Raton (2006)

12. Gasiński, L., Papageorgiou, N.S.: Anisotropic nonlinear Neumann problems. Calc. Var. Partial Differ. Equ. 42(3-4), 323-354 (2011)

13. Guedda, M., Véron, L.: Quasilinear elliptic equations involving critical Sobolev exponents. Nonlinear Anal. 13(8), 879-902 (1989)

14. Guo, Z., Zhang, Z.: $W^{1, p}$ versus $C^{1}$ local minimizers and multiplicity results for quasilinear elliptic equations. J. Math. Anal. Appl. 286(1), 32-50 (2003)

15. Iannizzotto, A., Papageorgiou, N.S.: Existence of three nontrivial solutions for nonlinear Neumann hemivariational inequalities. Nonlinear Anal. 70(9), 3285-3297 (2009) 
16. Khan, A.A., Motreanu, D.: Local minimizers versus $X$-local minimizers. Optim. Lett. (2012). doi:10.1007/s11590-012-0474-8

17. Lieberman, G.M.: Boundary regularity for solutions of degenerate elliptic equations. Nonlinear Anal. 12(11), 1203-1219 (1988)

18. Lieberman, G.M.: The natural generalization of the natural conditions of Ladyzhenskaya and Ural'tseva for elliptic equations. Commun. Partial Differ. Equat. 16( 2-3), 311-361 (1991)

19. Mawhin, J., Willem, M.: Critical Point Theory and Hamiltonian Systems. Applied Mathematical Sciences, vol. 74. Springer-Verlag, New York (1989)

20. Miyajima, S., Motreanu, D., Tanaka, M.: Multiple existence results of solutions for the Neumann problems via super- and sub-solutions, J. Funct. Anal. 262(4), 1921-1953 (2012)

21. Motreanu, D., Papageorgiou, N.S.: Multiple solutions for nonlinear elliptic equations at resonance with a nonsmooth potential. Nonlinear Anal. 56(8), 1211-1234 (2004)

22. Motreanu, D., Papageorgiou, N.S.: Multiple solutions for nonlinear Neumann problems driven by a nonhomogeneous differential operator. Proc. Amer. Math. Soc. 139(10), 3527-3535 (2011)

23. Motreanu, D., Tanaka, M.: Generalized eigenvalue problems of nonhomogeneous elliptic operators and their application. Pacific J. Math. 265(1), 151-184 (2013)

24. Motreanu, D., Winkert, P.: On the Fučik spectrum for the $p$-Laplacian with Robin boundary condition. Nonlinear Anal. 74(14), 4671-4681 (2011)

25. Motreanu, D., Winkert, P.: The Fučík spectrum for the negative $p$-Laplacian with different boundary conditions. Nonlinear Analysis. Springer Optimization and Its Applications, vol.68, pp. 471-485. Springer, New York (2012)

26. Motreanu, D., Motreanu, V.V., Papageorgiou, N.S.: Nonlinear Neumann problems near resonance. Indiana Univ. Math. J. 58(3), 1257-1279 (2009)

27. Motreanu, D., Motreanu, V.V., Papageorgiou, N.S.: Multiple constant sign and nodal solutions for nonlinear Neumann eigenvalue problems. Ann. Scuola Norm. Sup. Pisa Cl. Sci. (5). 10(3), 729-755 (2011)

28. Motreanu, D., Motreanu, V.V., Papageorgiou, N.S.: On $p$-Laplace equations with concave terms and asymmetric perturbations. Proc. Roy. Soc. Edinb. A. 141A (1), 171-192 (2011)

29. Pucci, P., Serrin, J.: The Maximum Principle. Birkhäuser, Basel (2007)

30. Vazquez, J.L.: A strong maximum principle for some quasilinear elliptic equations. Appl. Math. Optim. 12(3), 191-202 (1984)

31. Winkert, P.: Constant-sign and sign-changing solutions for nonlinear elliptic equations with Neumann boundary values. Adv. Differ. Equat. 15(5-6), 561-599 (2010)

32. Winkert, P.: Local $C^{1}(\bar{\Omega})$-minimizers versus local $W^{1, p}(\Omega)$-minimizers of nonsmooth functionals. Nonlinear Anal. 72(11), 4298-4303 (2010)

33. Winkert, P., Zacher, R.: A priori bounds of solutions to elliptic equations with nonstandard growth. Discret. Contin. Dyn. Syst. Ser. S 5(4), 865-878 (2012) 